\title{
Graph theory reveals dysconnected hubs in 22q11DS and altered nodal efficiency in patients with hallucinations
}

\section{Marie-Christine Ottet ${ }^{1,2}$, Marie Schaer ${ }^{1,3}$, Martin Debbané ${ }^{1,4}$, Leila Cammoun ${ }^{2}$, Jean-Philippe Thiran ${ }^{2}$ and Stephan Eliez ${ }^{1,5}$}

\author{
Departement of Psychiatry, Office Médico-Pédagogique (OMP), University of Geneva School of Medicine, Geneva, Switzerland \\ 2 Signal Processing Laboratory (LTS5), Swiss Federal Institute of Technology (EPFL), Lausanne, Switzerland \\ ${ }^{3}$ Department of Psychiatry, Stanford Cognitive and System Neuroscience Laboratory, Stanford University, Palo Alto, CA, USA \\ ${ }^{4}$ Adolescence Clinical Psychology Research Unit, University of Geneva, Geneva, Switzerland \\ ${ }^{5}$ Department of Genetic Medicine and Development, University of Geneva School of Medicine, Geneva, Switzerland
}

\section{Edited by:}

Yong He, Beijing Normal University, China

\section{Reviewed by:}

Qingbao Yu, The Mind Research

Network, USA

Alex Fornito, University of

Melbourne, Australia

\section{${ }^{*}$ Correspondence:}

Marie-Christine Ottet, Department of Psychiatry, Office

Médico-Pédagogique, University of Geneva School of Medicine, Rue

David-Dufour 1, Case postale 50 1211 Geneva 8, GE, Switzerland e-mail:marie-christine.ottet@ unige.ch
Schizophrenia is postulated to be the prototypical dysconnection disorder, in which hallucinations are the core symptom. Due to high heterogeneity in methodology across studies and the clinical phenotype, it remains unclear whether the structural brain dysconnection is global or focal and if clinical symptoms result from this dysconnection. In the present work, we attempt to clarify this issue by studying a population considered as a homogeneous genetic sub-type of schizophrenia, namely the $22 q 11.2$ deletion syndrome (22q11.2DS). Cerebral MRIs were acquired for 46 patients and 48 age and gender matched controls (aged 6-26, respectively mean age $=15.20 \pm 4.53$ and $15.28 \pm 4.35$ years old). Using the Connectome mapper pipeline (connectomics.org) that combines structural and diffusion MRI, we created a whole brain network for each individual. Graph theory was used to quantify the global and local properties of the brain network organization for each participant. A global degree loss of $6 \%$ was found in patients' networks along with an increased Characteristic Path Length. After identifying and comparing hubs, a significant loss of degree in patients' hubs was found in $58 \%$ of the hubs. Based on Allen's brain network model for hallucinations, we explored the association between local efficiency and symptom severity. Negative correlations were found in the Broca's area $(p<0.004)$, the Wernicke area $(p<0.023)$ and a positive correlation was found in the dorsolateral prefrontal cortex (DLPFC) $(p<0.014)$. In line with the dysconnection findings in schizophrenia, our results provide preliminary evidence for a targeted alteration in the brain network hubs' organization in individuals with a genetic risk for schizophrenia. The study of specific disorganization in language, speech and thought regulation networks sharing similar network properties may help to understand their role in the hallucination mechanism.

Keywords: DTI, small-world, network, Broca, psychosis, schizophrenia, human connectome, Wernicke

\section{INTRODUCTION}

22q11.2 deletion syndrome (22q11.2DS), also known as velocardio-facial syndrome (Shprintzen et al., 1978), is a wellestablished neurogenetic model for studying the pathogenesis of schizophrenia (Bassett and Chow, 1999). The prevalence rate of the 22q11DS population for developing schizophrenia is about $30 \%$, making it the third highest risk rate after having an affected monozygotic twin $(50 \%$ risk) or both parents being affected (46\% risk) (McGuffin et al., 1995). Furthermore, $30-50 \%$ of nonschizophrenic 22q11DS individuals demonstrate sub-threshold symptoms of psychosis (Feinstein et al., 2002). Considering the genetic 22q11.2DS model as a homogenous sub-type may highlight the presence of neurodevelopmental biomarkers underlining the schizophrenic disorders.

As schizophrenia is a heterogeneous disorder previous literature on white matter has revealed highly variable alterations throughout the brain and a few replicated findings (see Fitzsimmons et al., 2013 for a recent review). Due to confounding factors such as duration of illness, medication, age sample or methodology, it remains unclear whether schizophrenia demonstrates localized alterations or a whole brain dysconnection. Graph theory provides promising tools to analyze both whole brain phenomenon using global network measurements, and specific properties using local network measurements (Bassett and Bullmore, 2009; He and Evans, 2010; Rubinov and Sporns, 2010). Few studies in schizophrenia have used the graph theory in structural magnetic resonance imaging. Bassett et al. (2008) characterized the cerebral gray matter volumetric covariation in a large sample (>200) of patients with schizophrenia compared to healthy controls. Although the small-world properties were preserved, a reduced network hierarchy with a loss of hubs was found in individuals with schizophrenia and more specifically in frontal (bilateral dorsolateral prefrontal cortex) areas (Bassett et al., 2008). Graph theory was also used in studies of functional connectivity in schizophrenia. Evidence of global rather than focal functional dysconnectivity grows in schizophrenia 
literature. Disruption of the small-world properties has been found in patients (Liu et al., 2008) as well as weaker connectivity and lower clustering coefficient (Yu et al., 2012), and hubs alteration (Yu et al., 2012, 2013). The functional connection of several specific regions has also been found such as the temporal lobe, the parietal lobe, the thalamus, hippocampus but more interestingly, functional integration between sub-networks (such as the semantic network, the default network) is impaired (see Zhang et al., 2012; Alexander-Bloch et al., 2013). Using diffusion images for reconstructing brain networks, van den Heuvel et al. (2010) replicated the observation that individuals with schizophrenia have preserved small-world properties and increased path length in frontal, temporal and occipital areas. Reduction of frontal hubs has also been demonstrated, involving the superior frontal and the anterior cingulate (van den Heuvel et al., 2010). Following Latora and Marchiori's conception of global and local efficiency measuring how well information is exchanged over the network (Latora and Marchiori, 2001), Wang et al. (2011) demonstrated that individuals with schizophrenia have a reduced global efficiency [reduced after controlling for the effect of age, gender and brain size (Wang et al., 2011)]. However, it remains unclear whether the network alterations are caused by the emergence of the schizophrenia disorder or if there is a predetermined network configuration that acts as a vulnerability factor for the later development of schizophrenia.

Amongst all the psychotic symptoms in the 22q11DS, hallucinations are often considered the most clinically salient signs of risk for psychosis (Debbané et al., 2006). Moreover, hallucinations constitute a valid early risk indicator for the development of schizophreniform disorders during adulthood (Poulton et al., 2000). As complex cognitive functions rely on a cerebral network involving several regions, (Sporns, 2010; Bassett and Gazzaniga, 2011) dysfunctions such as hallucination may result from abnormal topological connectivity between these areas (Lo et al., 2011). Structural and functional studies in individuals with schizophrenia suggest that several key regions play a role in the apparition of hallucinations and their severity [(see Allen et al., 2008) for a review]. In schizophrenic patients, reduction in the superior temporal gyrus (STG) gray matter volume has been associated with the severity of hallucinations (Flaum et al., 1995; Gaser et al., 2004; Onitsuka et al., 2004). Loss of gray matter volume in Broca's area has also been associated with this symptom (Gaser et al., 2004; Sumich et al., 2005). Several other brain areas, such as the insula (Shapleske et al., 2002), the thalamus (Neckelmann et al., 2006) and the supramarginal gyrus (Gaser et al., 2004), have been associated with the presence of hallucinations but have failed to show consistent volumetric reduction. Two network studies have explored the relationship between clinical symptoms of schizophrenia and brain network properties. Although van den Heuvel et al. (2010) found no significant association between the Positive and Negative Symptoms Scale (PNASS) (Kay et al., 1987) and topological features, Wang et al. (2011) showed a negative correlation between the PANSS (positive, negative and total scores) and global and local efficiency, meaning that the more severe the symptoms, the lower are both the local and the global topological efficiencies. Lower global efficiency and longer path length has also been related to higher score on the negative PANSS scale (Yu et al., 2011a,b).

The purpose of this present work is to study the global and local network features in a population at high genetic risk for schizophrenia (22q11.2DS) by focusing on the hierarchical structure of the brain network (hub topological configuration). Furthermore, we aim to explore the specific relationship between hallucination symptoms and the local efficiency of the related brain areas. According to Allen's model of brain regions involved in hallucinations (Allen et al., 2008), we suggest that the topological connectivity of the following regions-DLPFC, dorsal anterior cingulate, Broca's area, ventral anterior cingulate, orbitofrontal gyrus, and STG-will be associated with the severity of hallucinations in schizophrenia.

\section{MATERIALS AND METHODS PARTICIPANTS}

All the participants underwent the same protocol, which included an MRI session for collecting a structural T1-weigthed image and a diffusion image along with an IQ measure with the Wechsler Intelligence Scale for Children-Third Edition revised (Wechsler, 1991) or the Wechsler Adult Intelligence Scale-III for adults (Wechsler, 1997). The participant or their parents signed consent forms containing information about the study and its purpose. The detailed protocol of the study was previously reviewed and accepted by the Institutional Review Board of Geneva University School of Medicine.

\section{2q11.2DS GROUP}

Forty-six participants with a 22q11.2DS aged between 6 and 26 (mean $=15.20 \pm 4.53$ ), (23 males and 22 females) were recruited through parent associations in French speaking European countries. The 22q11.2 deletion was confirmed by a blood sample analyzed with the Quantitative Fluorescent Polymerase Chain Reaction (QF-PCR) performed on the deleted region. The average IQ was of $77.5 \pm 16.6$. All the patients were assessed by an experienced psychiatrist using the Brief Psychiatric Rating Scale (BPRS) (Leucht, 2005), the Diagnostic Interview for children and adolescents (DICA) (Reich, 2000) and the Structured Clinical Interview for DSM-IV AXIS I Disorders for adults (SCID) (First et al., 1996). Only one patient fulfilled the criteria for schizophrenia. The average BPRS hallucination subscale for the forty-six participants was $1.63 \pm 1.12$ and among them fourteen individuals reported to have verbal hallucination.

\section{CONTROL GROUP}

The participants from the control group were recruited among the siblings of the patients and in the community. The 48 control participants comprised 25 males and 24 females, aged from 7 to $24($ mean $=15.28 \pm 4.35)$. The average IQ was of $107 \pm 18.12$. None of the controls had present or past history of psychiatric or neurological disorders.

\section{MRI CHARACTERISTICS}

Using a Siemens Trio 3 Tesla scanner, we acquired a set of two cerebral MRIs for each participant. A T1-weighted sequence with a $3 \mathrm{D}$ volumetric pulse was collected using the following 
sequence: $T R=2500 \mathrm{~ms}, T E=3 \mathrm{~ms}$, flip angle $=8^{\circ}$, acquisition matrix of $256 \times 256$, field of view $=22 \mathrm{~cm}$, slice thickness $=1.1 \mathrm{~mm}, 192$ slices. The second MRI was a Diffusion Tensor Imaging (DTI) with the following parameters: number of directions $=30, b=1000 \mathrm{~s} / \mathrm{mm}^{2}, T E=82 \mathrm{~ms}, T R=[8300-8800] \mathrm{ms}$, flip angle $=90^{\circ}$, acquisition matrix of $128 \times 128$, field of view $25.6 \mathrm{~cm}$, slice thickness $=2 \mathrm{~mm}$.

\section{IMAGE PROCESSING}

The two acquired scans were processed for each participant using the Human Connectome Mapper (http://connectomics. org, Daducci et al., 2012). The software is a pipeline of several other software programs, combining each dedicated package for the purpose of creating an individual's connectome. For the T1-weigthed image, FreeSurfer software starts to remove all nonbrain tissue, segmenting the image in order to extract the white matter, the sub-cortical gray matter volume and the cortical surface (Dale et al., 1999; Fischl et al., 1999). This step is performed using both intensity and continuity data through the whole $3 \mathrm{D}$ volume. The surfaces and volumes generated have been validated against histological studies (Rosas et al., 2002). However, these automatic steps need verification and manual correction if necessary. At the end of this process, three-dimensional volumes or surfaces and a cortical segmentation are available For the diffusion images, first we use a correction for the effect of head motion and distortion of eddy currents through an affine alignment using the FLIRT tool of the FSL-FDT software (Jenkinson and Smith, 2001). The realigned images are used to reconstruct the white matter macroscopic bundles using the streamline deterministic tractography of Diffusion Toolkit (http://trackvis.org/). The registration of the T1-weighted onto the diffusion images is an affine transformation using the intensity-based linear registration tool FLIRT. When combined, the intersection of the estimated fibers and the segmented regions creates a connectome which is represented by the connection matrix.

\section{NETWORK MEASURES}

Graph theory describes the human connectome as a network of nodes (in our case cortical regions) and edges (in our case white matter bundles) connecting two nodes. This network can be described either with weighted edges (where edges contain the information about the strength of the two nodes) or with binary edges (where only the existence of a link is represented). Therefore, two kinds of measurements are applicable: measurements on a binary network or on a weighted network. Following the purpose of the present study, which is to try to delineate the core organization of individuals with 22q11.2DS, we decided to explore the configuration of the binary network. Furthermore, binary network analyses have the advantage of showing a low variability in the network measures (Cheng et al., 2012).

In the present study, we used the tools for measuring network properties included in the brain connectivity toolbox developed for Matlab (https://sites.google.com/site/bctnet/) (see Rubinov and Sporns, 2010) for the description and mathematical formula of each measure. The first measurement step is the analysis of the global characteristics of the patients' and controls' networks, using the Characteristic Path Length, the Mean Clustering
Coefficient, the Global Transitivity, the Global Efficiency and the Global Degree. In the context of the binary networks, the Global Degree represents the total number of edges (existing connections between two nodes) in the network. The Mean Clustering Coefficient measures the potential for functional segregation of the network and is calculated as the mean of the clustering coefficient, which is the fraction of the number of neighbors of a node that are also neighbors of each other (Watts and Strogatz, 1998). The Characteristic Path Length represents the average of the short path lengths of the network. The short path length is the number of edges that have to be crossed to go from one node to another. The Characteristic Path Length therefore measures the functional integration potential of a network. The normalized ratio between the Mean Clustering Coefficient and the Characteristic Path Length of a network gives the Smallworldness measure of the network (Watts and Strogatz, 1998; Humphries and Gurney, 2008). The Smallworldness measures the optimality between rapid communication throughout the network (functional integration) and the capacity to process locally based information (functional segregation) (Sporns and Honey, 2006). The optimal balance between the Characteristic Path Length and the Mean Clustering Coefficient can also be estimated by the Global Efficiency and the local efficiency (Latora and Marchiori, 2001). The Global and local efficiency measure how efficiently information is exchanged over the network, and respectively play similar roles to the Characteristic Path Length and the clustering coefficient.

The second analysis focuses on the hub configuration by ranking all of the nodes in the healthy control network on three local network measures, the local degree, the clustering coefficient and the betweenness centrality. The local degree measures a node's number of edges or neighbors. The clustering coefficient highlights a node's surrounding configurations by analyzing how many of its neighbors are also connected to each other. The betweenness centrality measures how many short path lengths pass through the node and is therefore a measure of node influence on the network (Rubinov and Sporns, 2010). By definition, the 20\% highest ranking nodes for all 3 values are considered to be the network hubs (Sporns et al., 2007; Sporns, 2010; van den Heuvel et al., 2010; van den Heuvel and Sporns, 2011). The analysis then continues by comparing the hubs and node degrees between the 22q11.2DS group and the healthy control group.

The third step consists of exploring the relationship between Allen's model's network characteristics (local efficiency) and the clinical measures such as the presence and severity of hallucinations (BPRS). According to Allen's model of hallucinations, the DLPFC, dorsal anterior cingulate, Broca's area, ventral anterior cingulate, orbitofrontal gyrus, the supplementary motor area and STG are the brain regions involved in hallucinations. These regions are not represented in the same way in the Freesurfer Desikan parcellation scheme: region that corresponds the most to the DLPFC is the rostral middle frontal parcel; for the superior temporal gyrus it is the superior temporal parcel and the transverse temporal parcel; for the ventral and dorsal anterior cingulated it is respectively the rostral anterior parcel and the caudal anterior cingulate parcel; the inferior frontal gyrus including Broca's area refers to the pars triangularis parcel, the pars 
opercularis parcel and the pars orbitalis parcel; and the orbito frontal gyrus corresponds to the lateral orbito-frontal parcel and the medial orbito-frontal parcel (see Figure 1). We decided not to include the supplementary motor area, as it is equally spread over three Freesurfer's regions (the caudal middle frontal, the superior frontal and the precentral).

As Latora and Marchiori (2001) demonstrated, the local efficiency measures the functional segregation of one node when this particular node is removed from the sub-network. This measure represents the level of local information-processing. More precisely the local efficiency measures the functional segregation which means the capacity of locally processed information. In the case of hallucination, Allen et al. postulated a neuroanatomical model, composed of several cortical regions where their dysfunctional interplay fosters the hallucination emergence. Therefore, we wanted to see if the local capacity of this sub-network to process the information was related to the emergence and the severity of the hallucination.

In order to analyze the efficiency of communication in Allen's theoretical network, we compared the local efficiency value of each node between patients and healthy participants. Then, in the patients' networks, we analyzed the relationship between each node's efficiency and the BPRS hallucination subscale. For the ten regions, an outlier analysis was applied on the efficiency

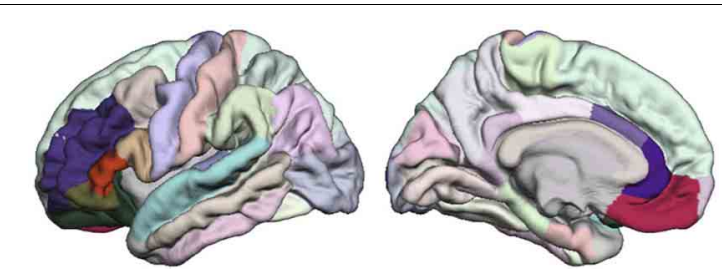

FIGURE 1 | Among the Desikan parcellation scheme, the regions elected as similar to Allen's model areas are the represented in full color and the remainder parcels are in faded color. On the lateral view: the superior temporal is cyan, the rostral middle frontal is purple, the lateral orbitofrontal is dark green, the pars orbitalis is khaki, the pars triangularis is dark orange, and the pars opercularis is beige. On the medial view, the medial orbitofrontal is fuchsia, the rostral anterior cingulate is dark purple, and the caudal cingulate is parme. measurement and the correlation was controlled for age and gender.

\section{SIMULATION METHOD}

As previous literature in the 22q11.2DS demonstrated that the brains of patients with 22q11.2DS show a $10 \%$ volumetric reduction (Eliez et al., 2000; Kates et al., 2001), which has an impact on the number of fibers ( $\sim 10 \%$ less fibers) (Ottet et al., 2013), in the current study we simulated a random $10 \%$ reduction on the controls' connectome. This simulation enables a determination of whether the network measures applied for comparing both groups are biased by the reduction of the number of fibers or not. Using Matlab, we randomly subtracted one fiber at a time until $10 \%$ of the total number of fibers in the network were removed. This procedure was replicated for each healthy control connectome before applying the global network measurements.

\section{RESULTS}

\section{GLOBAL NETWORK MEASURES}

All the global network results comparing patients, controls and simulated networks can be found in Table $\mathbf{1}$.

The one tailed $t$-test comparison between the number of fibers contained in the patients' network and the controls' network revealed a significant loss of $10 \%$ of fibers $(p<0.001)$. The same test was applied between the patients' and the simulated network revealing that the significance was no longer preserved ( $p=$ $0.59)$. Although the simulated network didn't show any difference in the number of fibers compared to the patient group, the number of edges demonstrated a connectivity reduction of $6 \%(p<$ 0.005). Similarly, the Global Efficiency was significantly reduced in the brain network of the patients $(p=0.0117)$. No difference was observed in the Mean Clustering Coefficient between the two groups. Although the Characteristic Path Length was increased for the patient group ( $p=0.04$ ), the Smallworldness measure did not differ significantly between the two groups $(p=0.368)$.

\section{HUB ANALYSIS}

To determine which of the 83 nodes are hubs in the healthy control network, each node was ranked on 3 local network measures: the local degree, the betweenness centrality and the clustering

Table 1 | Mean, standard deviation and significant differences in the brain network global measures for participants with 22q11.2DS, healthy participants and simulated networks.

\begin{tabular}{|c|c|c|c|c|c|c|c|c|}
\hline \multirow[t]{2}{*}{ Global network measures } & \multicolumn{2}{|c|}{ 22q11.2DS } & \multicolumn{2}{|c|}{ Control } & \multirow[t]{2}{*}{$\boldsymbol{p}$} & \multicolumn{2}{|c|}{ Simulated } & \multirow[t]{2}{*}{$p$} \\
\hline & Mean & Std & Mean & Std & & Mean & Std & \\
\hline Total number of fibers & 42087 & 6608 & 46764 & 7343 & 0.0005 & 42385 & 5098 & 0.59 \\
\hline Total number of edges & 1269.8 & 167.5 & 1354.5 & 132.4 & 0.0038 & 1354.5 & 132.4 & 0.0038 \\
\hline Characteristic path length & 2.1652 & 0.1640 & 2.1150 & 0.1069 & 0.0402 & 2.1150 & 0.1069 & 0.0402 \\
\hline Mean clustering coefficient & 0.7319 & 0.0295 & 0.7345 & 0.0234 & 0.3224 & 0.7345 & 0.0234 & 0.3224 \\
\hline Global efficiency & 0.5238 & 0.0335 & 0.5372 & 0.0221 & 0.0117 & 0.5372 & 0.0221 & 0.0117 \\
\hline Smallworldness & 1.6837 & 0.0859 & 1.68961 & 0.0783 & 0.368 & 1.68961 & 0.0783 & 0.368 \\
\hline
\end{tabular}

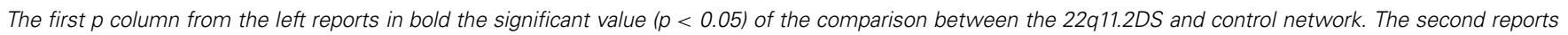
in bold the significant values ( $<0.05$ ) when comparing the 22q11.2DS with the simulated network. 


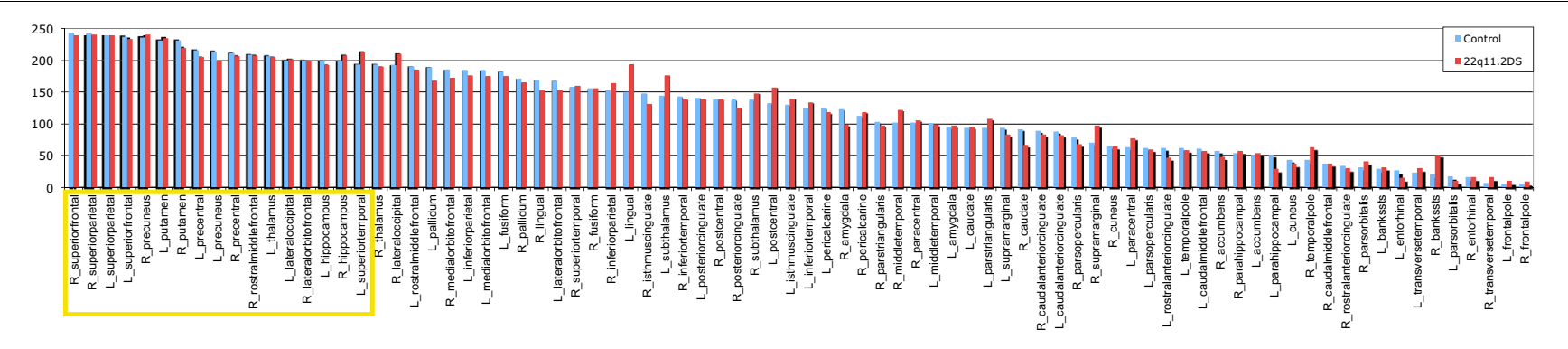

FIGURE 2 | Final ranking of the $\mathbf{8 2}$ gray matter regions in the healthy controls' brain. The 17 nodes with the highest rank highlighted in yellow, are considered as the connector hubs of the network. In blue is represented the repartition of the final ranking for the control and in red for the $22 \mathrm{q} 11.2 \mathrm{DS}$.

coefficient. According to the literature, hubs are defined as nodes that demonstrate a high degree, a high centrality and a low clustering coefficient. A ranking score was attributed to each of the nodes for the three measures previously discussed and averaged for the 48 healthy controls. The highest connected and the most central node scored 83 and the least connected and least central node scored 1 . Inversely the least clustered node scored 83 and the most clustered scored 1 . The final classification is the addition of the three scores, in which the top 20\% are considered as the connector hubs of the network (see yellow bars in Figure 2). Although the stem node was the highest node on the final classification, we did not consider it as a hub because it is not a gray matter region (therefore all subsequent analyses considered 82 regions and not 83 ). Twelve out of 17 hubs were represented on both hemispheres in the superior frontal, the hippocampus, the superior parietal, the precuneus, the precentral and the putamen. In the right hemisphere two supplementary hubs are found, the rostral middle frontal and the lateral orbito-frontal. In the left hemisphere, three supplementary hubs were found, the superior temporal the lateral occipital and the thalamus.

\section{DEGREE ANALYSIS}

The results demonstrated that 26 nodes out of $82(33 \%)$ in the patients' networks have a significantly reduced degree after FDR correction $(\mathrm{pFDR}=0.0149$ ). The names of the brain regions affected are listed in Table 2. Only one node showed an increased degree in the patients' networks: the right supramarginal region.

When analyzing how many of the hubs are altered, we noted that 10 out of 17 hubs (58\%) were reduced in the patients network (see Figure 3). Conversely only 16 non-hubs out of 65 (25\%) were reduced (see Table 2 ). Therefore, the percentage of affected hubs is more than double that of affected non-hub nodes. The 10 affected hubs are the bilateral hippocampus, superior parietal and precentral regions, right rostral middle frontal, superior frontal, precuneus and left thalamus.

\section{EFFICIENCY AND HALLUCINATIONS}

Following Allen's brain network model, the efficiency of ten nodes of the left hemisphere was correlated with the BPRS hallucination subscale. After controlling for age and gender, three of them showed a significant association with the presence and/or severity of the symptoms. Both the pars triangularis parcel and the transverse temporal parcel demonstrated a negative correlation with

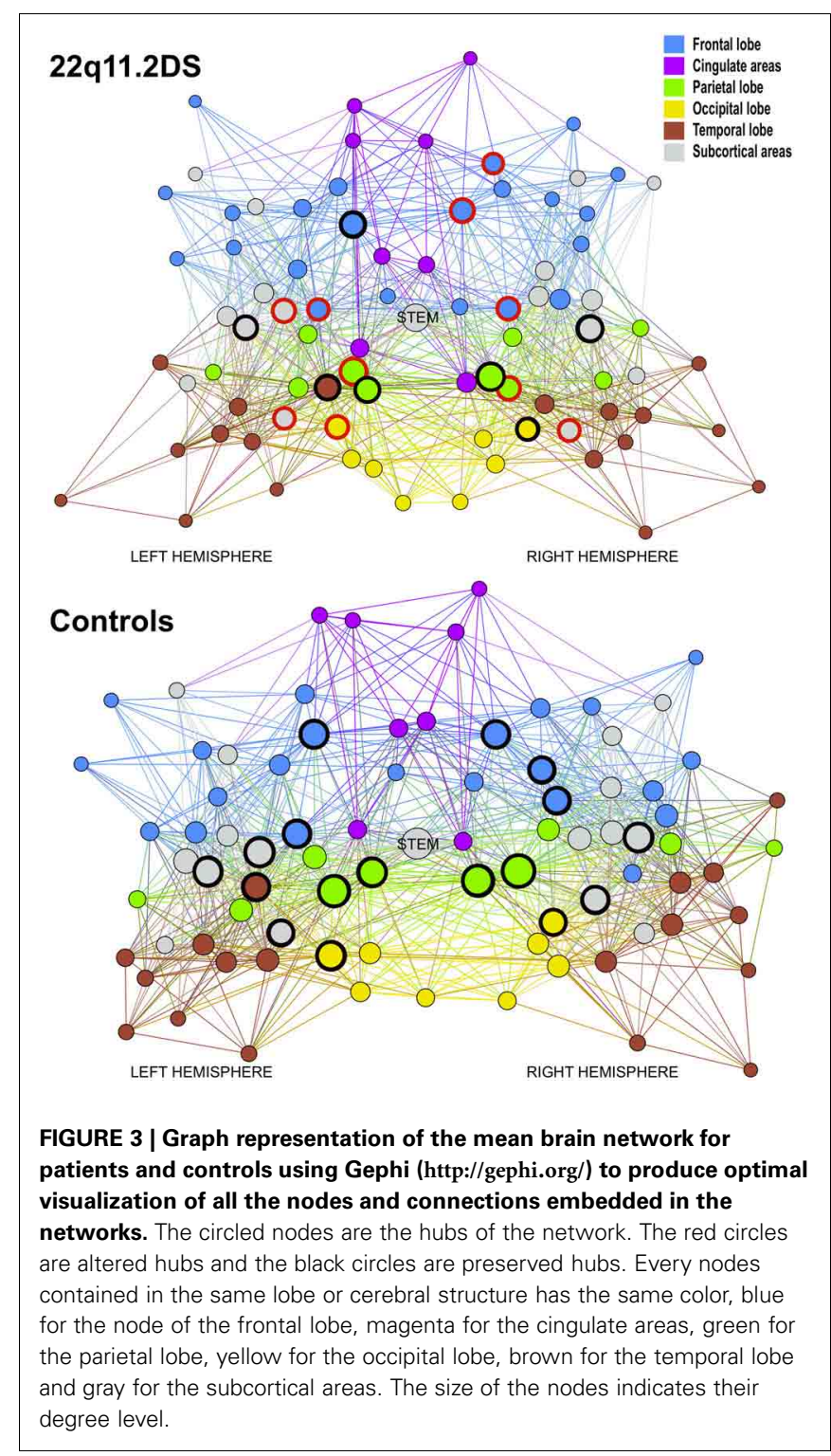

the symptom's scale (respectively $R=-0.312, p=0.04$, uncorrected and $R=-0.354, p=0.023$, uncorrected). Inversely, the rostral middle frontal shows a positive correlation with the symptom's scale ( $R=0.373, p=0.014$, uncorrected) (see Figure 4). 
Table 2 | List of the 26 nodes out of 82 that are significantly different in the patients' network after FDR correction, and indication as being hubs or not.

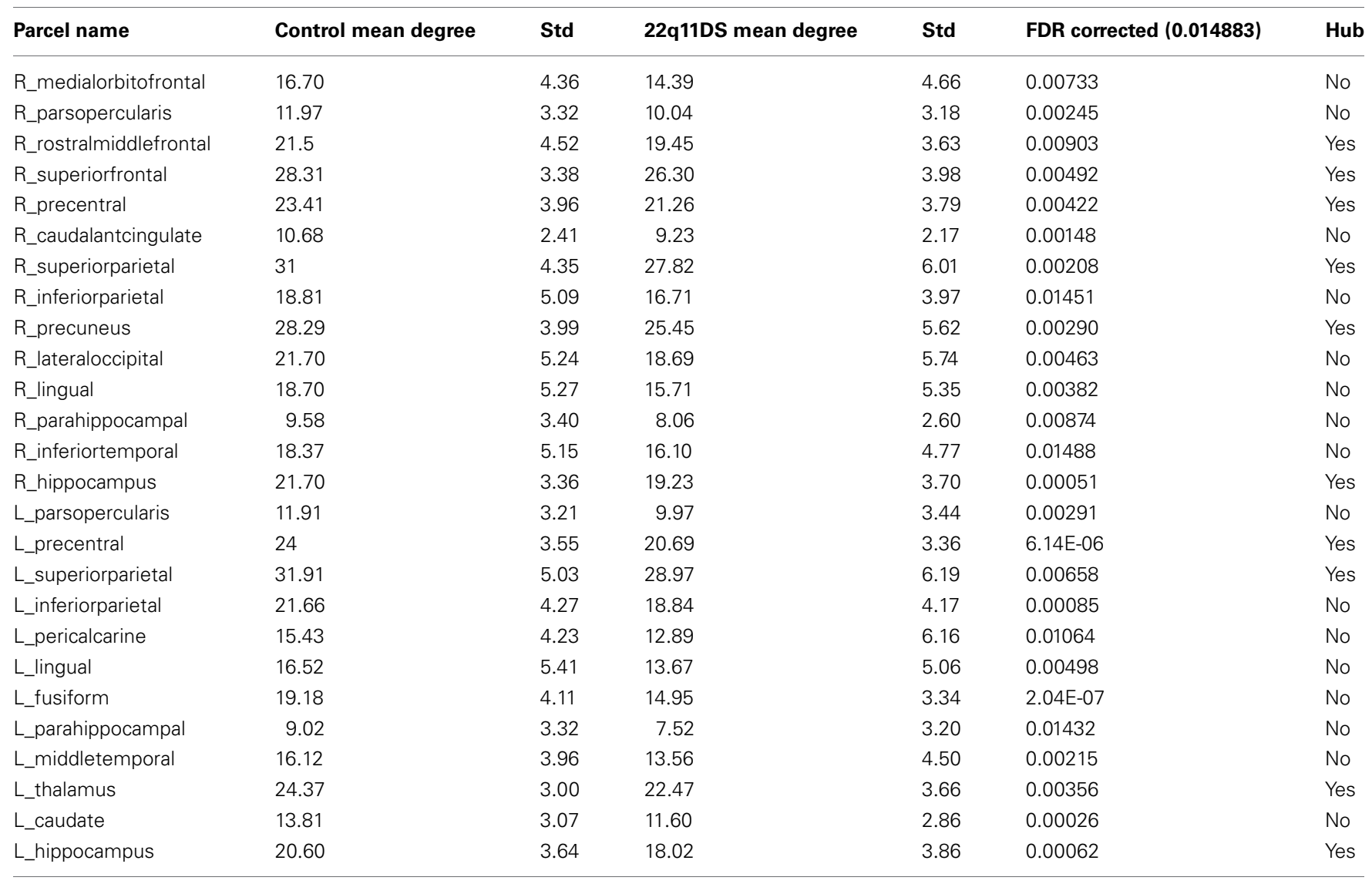

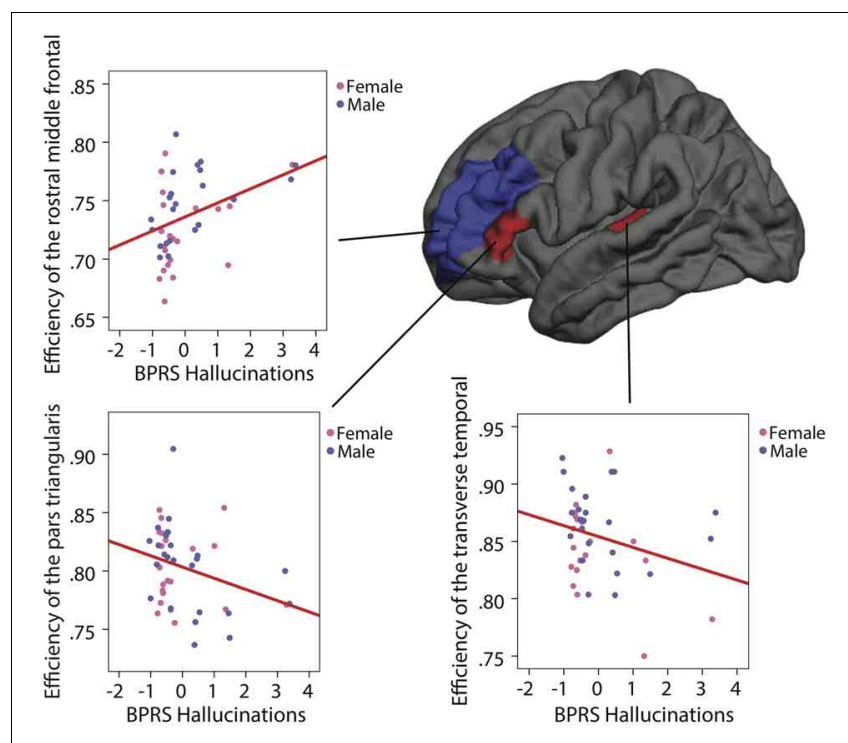

FIGURE 4 | Correlations between the BPRS hallucination subscale and the network efficiency in individuals with 22q11.2DS after age and gender correction. On the left hemisphere, the red regions (pars triangularis and transverse temporal) represent a negative correlation and the blue region (rostral middle frontal) represents a positive correlation.
The level of IQ, an additional controlling variable, was introduced, but nevertheless the significance for the three correlations survived.

\section{DISCUSSION}

Using white matter deterministic tractography and gray matter surface-based parcellation to reconstruct the brain connectome, the present work is the first study that analyzes the brain connectome in the 22q11.2DS in the light of the graph theory framework. In line with previous white matter studies in the 22 q11DS, we found a $10 \%$ reduction in the number of fibers in the brain connectome of patients with 22q11DS (Eliez et al., 2000; Kates et al., 2001; Gothelf et al., 2011; Ottet et al., 2013). We also observed a $6 \%$ reduction of connectivity in patients, which means that $6 \%$ of the edges of the patients' network were missing. The simulation analysis demonstrated that the initial $10 \%$ fiber loss was not the cause of the $6 \%$ connectivity loss. This global dysconnection found in our analyses sustains the dysconnection hypothesis in schizophrenia (Friston and Frith, 1995; Stephan et al., 2009).

Despite the observed global disconnection, graph theoretical analysis comparing individuals with a high risk of developing schizophrenia (22q11.2DS) and healthy controls revealed that the smallworldness property of the patients' brain network was still 
preserved. However, a longer path length, similar to a lower global efficiency, demonstrates that the functional integration [capacity to transmit information more directly, with less interference or attenuation (Latora and Marchiori, 2001)] is reduced in 22q11.2 deletion syndrome. Although the global clustering coefficient is not significantly different from the healthy controls, the smallworld brain organization of patients with 22q11.2DS tends to be closer to a regular network organization, in which the functional segregation (capacity to process specialized information, organized in clusters) is preserved but the functional integration is not optimum. Our study is in line with the previous network analysis on non-syndromic schizophrenia patients where a longer Characteristic Path Length has been systematically found (van den Heuvel et al., 2010; Zalesky et al., 2010b; Wang et al., 2011).

In the present work, local connectivity analysis allowed localization of the network nodes that were significantly altered in the patients' network. Every lobe and sub-cortical structure had one or more disconnected nodes sustaining the hypothesis that there is a widespread impact on the brain network in schizophrenia (Fornito et al., 2012). However, among the nodes that had a lower connectivity in patients, the proportion of affected hubs compared to non-affected hubs $(58 \%)$ is interesting. Similarly in schizophrenia, previous findings shows a loss of hub connectivity specifically in frontal lobes (van den Heuvel et al., 2010). Our finding shed light on a possible targeted alteration of cerebral hubs in the 22q11.2DS that may also have some relevance for schizophrenia. Network hubs have special integrative or control functions as their privileged position in the hierarchical organization is postulated to be a key element for large-scale cognitive abilities. Because of their high centrality and influence any perturbation in a hub would heavily impact brain network function (Sporns et al., 2007). The major hub connectivity alteration in individuals with 22q11.2DS may explain their mild cognitive impairments, but also the cognitive collapse seen in schizophrenia during and after their first psychotic episode.

Schizophrenia is a disease with multifactorial etiologies. However, among the multiple causes, genetic factors play an important role (Stephan et al., 2006). Studying brain alterations in populations with high risk or ultra-high risk of developing schizophrenia could break out the predetermining cerebral organization leading to the development of psychosis (Cannon et al., 2007). To the best of our knowledge, no study to date has measured the network properties of white matter connectivity using graph theory in adolescents at risk for schizophrenia. However, a recent study by Shi et al. (2012) demonstrates a significant reduction of global efficiency, an increased global Characteristic Path Length, less hub nodes and lower edge "global efficiency" in neonates' brain networks having a mother with schizophrenia or a schizoaffective disorder. These results suggest that the topological abnormalities in individuals carrying a familial genetic risk for schizophrenia can already be observed a few weeks after birth (Shi et al., 2012). As the 22q11.2 deletion syndrome is commonly considered as a high risk population for schizophrenia (Bassett and Chow, 1999), our study adds some evidence to the hypothesis that early alterations in a cerebral network organization due to genetic factors may partially drive the development of schizophrenia and psychotic symptoms such as hallucinations.
Indeed, the graph theory of large-scale brain networks postulates that cognitive abilities arise from several cerebral regions interacting together (Bressler and Menon, 2010). In our graph network study, we explored the relationship between the alterations of a topological network property and a cognitive dysfunction. Our analyses revealed that the local efficiency value within three parcels of the left hemisphere, namely Broca's area (pars triangularis), Wernicke's area (transverse temporal) and the dorsolateral prefrontal cortex (DLPFC) (rostral middle frontal), correlated significantly with clinical ratings of hallucination severity. Efficiency values in Broca and Wernicke's areas suggested that, as local connections within these parcels decreased, the severity of hallucinatory phenomena increased in our 22q11DS sample. Given the implication of these areas in language production and comprehension, our results suggest that impairments in the different components of language processing in 22q11DS may significantly contribute to the expression of hallucinations. These findings are consistent with previously reported associations between a decrease in verbal IQ and psychotic symptoms (Gothelf et al., 2005; Debbané et al., 2006), and further suggest that local network connectivity in key language areas of the brain contributes to hallucinations in this deletion syndrome (Gothelf et al., 2011).

Hallucination severity further correlated positively with efficiency within the dorsolateral prefrontal parcel. The DLPFC region is one of the most consistently examined regions in MRI studies involving individuals with schizophrenia (Lewis et al., 2004) because of its implications at different levels of impairments, from working memory and executive functions to dopaminergic system malfunction (Tanaka, 2006). Neuromodulation of the DLPFC activity and its role in regulation of thought content is hypothesized to depend on its connectivity patterns with surrounding regions (Arnsten et al., 2012). In this perspective, our results may suggest that an atypically high local efficiency in 22q11DS works against the DLPFC's connectivity with surrounding parcels that modulate its activity. This impairment may thus increase the propensity to experience hallucinations. Future functional MRI studies should examine the connectivity dynamics of the DLPFC more specifically to evaluate its contributions to symptoms such as hallucinations. Overall, our results add important information about the relevance of brain topological network organization to previous longitudinal investigations in 22q11DS that have linked cerebral integrity of gray matter in the DLPFC to the development of psychosis (Gothelf et al., 2005; Schaer et al., 2009; Gothelf et al., 2011). Further research is necessary to understand the maturational dynamics between gray and white matter, and how these may interact to increase the potential for psychotic symptoms in 22q11DS.

\section{LIMITATIONS}

In this study, Graph theory principles were applied for the first time on a population with 22q11.2DS bringing new insight on the alterations in their brain organization that could in turn lead to schizophrenia symptoms. Nevertheless, the present work shows some limitations.

Although we choose the recommended ratio between scan timing, voxel size and the number of directions, the same 
limitations found in every DTI and tractography study remain present. Listed in detail in (Bammer et al., 2003), the major limitations are the absence of in vitro validation studies and the distance-related effect of the tractography [see the limitation section in (Ottet et al., 2013)]. This distance related effect biases the number of fibers included in a bundle. Nevertheless, as the present work used unweighted (binary) network analysis, we do not take in consideration the number of fibers, therefore the latter issue is not expected to influence our analyses.

The comparison of brain networks when assessing their properties with graph theory measures, is very sensitive with regard to two constitutive elements, first the choice of the nodes and second the connection density. The first limitation relies on the choice of the cortical parcellation and its scale. Indeed, the choice of a network node is a critical step (He and Evans, 2010). Zalesky et al. (2010a,b) demonstrated the strong influence on network measures of different choices of parcellation scales and diffusion imaging, which impairs the comparability and the constituency across studies (Zalesky et al., 2010a). A wider discrepancy arises when comparing two different parcellation scales (80 vs. 4000 nodes) and/or when comparing two different diffusion imaging techniques [High angular (HARDI) diffusion vs. DTI]. The analyses in our study do not suffer from this issue as we compared our patients' cerebral diffusion image with the same sequence in healthy control cerebral images. Furthermore, for both patients and controls we used the same connectome processing pipeline including the parcellation scheme, which demonstrated a good to excellent test-retest reliability on graph network measures (Owen et al., 2013). Therefore, the comparison between the two populations of the global and local network measures does not suffer from this kind of issue.

Nevertheless, the Desikan parcellation scheme we chose for processing our participants' brain may yield an issue. Although this parcellation is based on the primary and secondary sulci delineation which confers a very high reliability across humans brains (Desikan et al., 2006), the size of the parcels differ importantly. Thus, when delineating the hubs of the network, the very large parcels display a higher degree value, which is one of the three measures used to rank the nodes onto the hub scale. This concern is valid for the two other measures used for delineating the hubs (clustering coefficient and the centrality). However, the hubs we found were highly consistent with previous literature. Indeed, Li et al. (2011) evaluated as the highest reliable hubs the

\section{REFERENCES}

Alexander-Bloch, A., Giedd, J. N., and Bullmore, E. (2013). Imaging structural co-variance between human brain regions. Nat. Rev. Neurosci. 14, 322-336. doi: 10.1038/nrn3465

Allen, P., Larøi, F., McGuire, P. K., and Aleman, A. (2008). The hallucinating brain: a review of structural and functional neuroimaging studies of hallucinations. Neurosci. Biobehav. Rev. 32, 175-191. doi: 10.1016/j.neubiorev.2007.07.012

Arnsten, A. F. T., Wang, M. J., and Paspalas, C. D. (2012).
Neuromodulation of thought: flexibilities and vulnerabilities in prefrontal cortical network synapses. Neuron 76, 223-239. doi: 10.1016/j.neuron.2012.08.038

Bammer, R., Acar, B., and Moseley, M. E. (2003). In vivo MR tractography using diffusion imaging. Eur. J. Radiol. 45, 223-234. doi: 10.1016/S0720-048X(02)00311-X

Bassett, A. S., and Chow, E. W. (1999). 22q11 deletion syndrome: a genetic subtype of schizophrenia. Biol. Psychiatry 46, 882-891. doi: 10.1016/S0006-3223(99)00114-6

bilateral putamen, bilateral superior frontal and left precuneus among three tractography methods (Li et al., 2011). van den Heuvel and Sporns (2011) found that bilaterally, the precuneus, superior frontal and superior parietal, hippocampus, putamen and thalamus were hubs of structural derived networks (van den Heuvel and Sporns, 2011). Amongst all the hubs delineated in our study, only the bilateral precentral could have been elected because of its large size.

The second limitation concerns the difference in the number of connections that exist between the control and patient networks. This discrepancy may bias the topological measurements and may result in the significant findings. As van Wijk et al. shows in 2010, there is no way to rule out these differences without introducing another bias.

\section{CONCLUSION}

In the present study, the targeted dysconnectivity of the hubs in a population considered as a model for schizophrenia (22q11.2DS) suggests the existence of an early alteration in the cerebral network organization that is due to genetic factors which may partially drive the development of schizophrenia and psychotic symptoms. Furthermore, altered local efficiency in areas responsible for language processing (Broca and Wernicke) sheds light on the implication of structural network organization in the severity of hallucinations. Further research is needed to understand the interaction between structural networks and psychotic symptoms.

\section{ACKNOWLEDGMENTS}

This research was funded by the Swiss National Research Fund, with funds to Dr. Stephan Eliez (3200-063135.00/1, 3232-063134.00/1, PP0033-102864 and 32473B-121996), and by the National Center of Competence in Research (NCCR) "SYNAPSY_-The Synaptic Bases of Mental Diseases" financed by the Swiss National Science Foundation (nu 51AU40_125759) and by the Center for Biomedical Imaging (CIBM) of the GenevaLausanne Universities and the EPFL, as well as the foundations Leenaards and Louis-Jeantet. The authors would also like to warmly thank all the participants and their family for volunteering in this study. We also greatly thank Sarah Menghetti for organizing and collecting data, and Jason Last for proof reading this manuscript. Our thank goes also to François Lazeyras, Frank Henri, Yohann Ouvrier-Buffet.

Bassett, D. S., and Bullmore, E. T. (2009). Human brain networks in health and disease. Curr. Opin. Neurol. 22, 340-347. doi: 10.1097/WCO.0b013e32832d93dd

Bassett, D. S., Bullmore, E., Verchinski, B. A., Mattay, V. S., Weinberger, D. R., and Meyer-Lindenberg, A. (2008). Hierarchical organization of human cortical networks in health and schizophrenia. J. Neurosci. 28, 9239-9248. doi: 10.1523/JNEUROSCI.1929-08.2008

Bassett, D. S., and Gazzaniga, M. S. (2011). Understanding complexity in the human brain. Trends Cogn. Sci. (Regul Ed) 15, 200-209. doi: 10.1016/j.tics.2011.03.006

Bressler, S. L., and Menon, V. (2010). Large-scale brain networks in cognition: emerging methods and principles. Trends Cogn. Sci. (Regul. Ed.) 14, 277-290. doi: 10.1016/j.tics. 2010.04.004

Cannon, T. D., Cornblatt, B., and McGorry, P. (2007). Introduction: the empirical status of the ultra high-risk (prodromal) research paradigm. 
Schizophr. Bull. 33, 661-664. doi: 10.1093/schbul/sbm031

Cheng, H., Wang, Y., Sheng, J., Kronenberger, W. G., Mathews, V. P., Hummer, T. A., et al. (2012). Characteristics and variability of structural networks derived from diffusion tensor imaging. Neuroimage 61, 1153-1164. doi: 10.1016/j.neuroimage.2012.03.036

Daducci, A., Gerhard, S., Griffa, A., Lemkaddem, A., Cammoun, L., Gigandet, X., et al. (2012). The connectome mapper: an open-source processing pipeline to map connectomes with MRI. PLOS ONE 7:e48121. doi: 10.1371/journal.pone.0048121

Dale, A. M., Fischl, B., and Sereno, M. I. (1999). Cortical surfacebased analysis i. segmentation and surface reconstruction. Neuroimage 194, 179-194. doi: 10.1006/nimg.1998.0395

Debbané, M., Glaser, B., David, M. K., Feinstein, C., and Eliez, S. (2006). Psychotic symptoms in children and adolescents with 22q11.2 deletion syndrome: neuropsychological and behavioral implications. Schizophr. Res. 84, 187-193. doi: 10.1016/j.schres.2006.01.019

Desikan, R. S., Ségonne, F., Fischl, B., Quinn, B. T., Dickerson, B. C., Blacker, D., et al. (2006). An automated labeling system for subdividing the human cerebral cortex on MRI scans into gyral based regions of interest. Neuroimage 31, 968-980. doi: 10.1016/j.neuroimage.2006.01.021

Eliez, S., Schmitt, J. E., White, C. D., and Reiss, A. L. (2000). Children and adolescents with velocardiofacial syndrome: a volumetric MRI study. Am. J. Psychiatry 157, 409-415. doi: 10.1176/appiajp.157. 3.409

Feinstein, C., Eliez, S., Blasey, C., and Reiss, A. L. (2002). Psychiatric disorders and behavioral problems in children with velocardiofacial syndrome: usefulness as phenotypic indicators of schizophrenia risk. Biol. Psychiatry 51, 312-318. doi: 10.1016/S0006-3223(01)01231-8

First, M. B., Spitzer, R. L., Gibbon, M., and Williams, J. B. W. (1996). Structured Clinical Interview for DSM-IV Axis I Disorders, Clinician Version (SCID-CV). Washington, D.C: American Psychiatric Press, Inc.

Fischl, B., Sereno, M. I., and Dale, A. M. (1999). Cortical surfacebased analysis ii.inflation flattening and a surface-based coordinate system. Neuroimage 207, 195-207. doi: 10.1006/nimg.1998.0396
Fitzsimmons, J., Kubicki, M., and Shenton, M. E. (2013). Review of functional and anatomical brain connectivity findings in schizophrenia. Curr. Opin. Psychiatry 26, 172-187. doi: 10.1097/YCO.0b013e32835d9e6a

Flaum, M., Oandapos;Leary, D. S., Swayze, V. W., Miller, D. D., Arndt, S., and Andreasen, N. C. (1995). Symptom dimensions and brain morphology in schizophrenia and related psychotic disorders. J. Psychiatr. Res. 29, 261-276. doi: 10.1016/0022-3956(94)00046-T

Fornito, A., Zalesky, A., Pantelis, C., and Bullmore, E. T. (2012). Schizophrenia, neuroimaging and connectomics. Neuroimage 62, 2296-2314. doi: 10.1016/j.neuroimage.2011.12.090

Friston, K. J., and Frith, C. D. (1995). Schizophrenia: a disconnection syndrome? Clin. Neurosci. 3, 89-97.

Gaser, C., Nenadic, I., Volz, H. P., Büchel, C., and Sauer, H. (2004). Neuroanatomy of "hearing voices": a frontotemporal brain structural abnormality associated with auditory hallucinations in schizophrenia. Cereb. Cortex 14, 91-96. doi: 10.1093/cercor/bhg107

Gothelf, D., Furfaro, J. A., Penniman, L. C., Glover, G. H., and Reiss, A. L. (2005). The contribution of novel brain imaging techniques to understanding the neurobiology of mental retardation and developmental disabilities. Ment. Retard. Dev. Disabil. Res. Rev. 11, 331-339. doi: 10.1002/mrdd.20089

Gothelf, D., Hoeft, F., Ueno, T., Sugiura, L., Lee, A. D., Thompson, P., et al. (2011). Developmental changes in multivariate neuroanatomical patterns that predict risk for psychosis in 22q11.2 deletion syndrome. J. Psychiatr. Res. 45, 322-331. doi: 10.1016/j.jpsychires.2010.07.008

He, Y., and Evans, A. (2010). Graph theoretical modeling of brain connectivity. Curr. Opin. Neurol. 23, 341-350. doi: 10.1097/WCO.0b01 3e32833aa567

Humphries, M. D., and Gurney, K. (2008). Network "small-worldness": a quantitative method for determining canonical network equivalence. PLoS ONE 3:e0002051. doi: 10.1371/journal.pone.0002051

Jenkinson, M., and Smith, S. (2001). A global optimisation method for robust affine registration of brain images. Med. Image Anal. 5, 143-156. doi: 10.1016/S1361-8415(01)00036-6

Kates, W. R., Burnette, C. P., Jabs, E. W., Rutberg, J., Murphy, A. M., Grados, M., et al. (2001). Regional cortical white matter reductions in velocardiofacial syndrome: a volumetric MRI analysis. Biol. Psychiatry 49, 677-684. doi: 10.1016/S00063223(00)01002-7

Kay, S. R., Fiszbein, A., and Opler, L. A. (1987). The positive and negative syndrome scale (PANSS) for schizophrenia. Schizophr. Bull. 13, 261-276. doi: 10.1093/schbul/ 13.2.261

Latora, V., and Marchiori, M. (2001). Efficient behavior of small-world networks. Phys. Rev. Lett. 87:198701 doi: $\quad 10.1103 /$ PhysRevLett.87. 198701

Leucht, S. (2005). Clinical implications of brief psychiatric rating scale scores. Br. J. psychiatry 187, 366-371. doi: 10.1192/bjp.187.4.366

Lewis, D. A., Cruz, D., Eggan, S., and Erickson, S. (2004). Postnatal development of prefrontal inhibitory circuits and the pathophysiology of cognitive dysfunction in schizophrenia. Ann. N.Y. Acad. Sci. 1021, 64-76. doi: 10.1196/annals.1308.008

Li, L., Rilling, J. K., Preuss, T. M., Glasser, M. F., and Hu, X. (2011) The effects of connection reconstruction method on the interregional connectivity of brain networks via diffusion tractography. Hum. Brain Mapp. 33, 1894-1913. doi: 10.1002/hbm.21332

Liu, Y., Liang, M., Zhou, Y., He, Y., Hao, Y., Song, M., et al. (2008). Disrupted small-world networks in schizophrenia. Brain 131, 945-961. doi: 10.1093/brain/awn018

Lo, C.-Y. Z., He, Y., and Lin, C. P. (2011). Graph theoretical analysis of human brain structural networks. Rev. Neurosci. 22, 1-13. doi: 10.1515/RNS.2011.039

McGuffin, P., Owen, M. J., and Farmer, A. E. (1995). Genetic basis of schizophrenia. Lancet 346, 678-682. doi: 10.1016/S0140-6736 (95) $92285-7$

Neckelmann, G., Neckelmann, D. A. G., Specht, K., Lund, A., Ersland, L., Smievoll, A. I., et al. (2006). MR Morphometry Ananlsysis of gray matter volume reduction in schizophrenia: association with hallucinations. Int. J. Neurosci. 116, 9-23. doi: 10.1080/0020745069096 2244

Onitsuka, T., Shenton, M. E., Salisbury, D. F., Dickey, C. C., Kasai, K., Toner, S. K., et al. (2004). Middle and inferior temporal gyrus gray matter volume abnormalities in chronic schizophrenia: an MRI study. Am. J. Psychiatry 161, 1603-1611. doi: 10.1176/appi.ajp 161.9.1603
Ottet, M.-C., Schaer, M., Cammoun, L., Schneider, M., Debbané, M., Thiran, J.-P., et al. (2013). Reduced fronto-temporal and limbic connectivity in the 22q11.2 deletion syndrome: vulnerability markers for developing schizophrenia? PLOS ONE 8:e58429. doi: 10.1371/journal. pone.0058429

Owen, J. P., Ziv, E., Bukshpun, P. Pojman, N., Wakahiro, M., Berman, J., et al. (2013). Test-retest reliability of computational network measurements derived from the structural connectome of the human brain. Brain Connect. 3, 160-176. doi: 10.1089/brain.2012. 0121

Poulton, R., Caspi, A., Moffitt, T E., Cannon, M., Murray, R., and Harrington, H. (2000). Children's self-reported psychotic symptoms and adult schizophreniform disorder: a 15-year longitudinal study. Arch. Gen. Psychiatry 57, 1053-1058. doi: 10.1001/archpsyc. 57.11 .1053

Reich, W. (2000). Diagnostic interview for children and adolescents (DICA). J. Am. Acad. Child Adolesc. Psychiatry 39, 59-66. doi: 10.1097/ 00004583-200001000-00017

Rosas, H. D., Liu, A. K., Hersch, S., Glessner, M., and Ferrante, R. J. (2002). Regional and progressive thinning of the cortical ribbon in Huntington' $s$ disease. Neurology 05886, 695-701. doi: 10.1212/WNL.58.5.695

Rubinov, M., and Sporns, O. (2010). Complex network measures of brain connectivity: uses and interpretations. Neuroimage 52, 1059-1069. doi: 10.1016/j.neuroimage.2009. 10.003

Schaer, M., Debbané, M., Bach Cuadra, M., Ottet, M.-C., Glaser, B., Thiran, J.-P., et al. (2009). Deviant trajectories of cortical maturation in 22q11.2 deletion syndrome (22q11DS): a crosssectional and longitudinal study. Schizophr. Res. 115, 182-190. doi: 10.1016/j.schres.2009.09.016

Shapleske, J., Rossell, S. L., Chitnis, X. A., Suckling, J., Simmons, A., Bullmore, E. T., et al. (2002). A computational morphometric MRI study of schizophrenia: effects of hallucinations. Cereb. Cortex 12, 1331-1341. doi: 10.1093/cercor/12. 12.1331

Shi, F., Yap, P.-T., Gao, W., Lin, W., Gilmore, J. H., and Shen, D. (2012). Altered structural connectivity in neonates at genetic risk for schizophrenia: a combined study using morphological and white 
matter networks. Neuroimage 62, 1622-1633. doi: 10.1016/j.neuro image.2012.05.026

Shprintzen, R. J., Goldberg, R. B., Lewin, M. L., Sidoti, E. J., Berkman, M. D., Argamaso, R. V., et al. (1978). A new syndrome involving cleft palate, cardiac anomalies, typical facies, and learning disabilities: velo-cardio-facial syndrome. Cleft Palate J. 15, 56-62.

Sporns, O. (2010). Networks of the Brain. MA: MIT Press. Available online at: http://books.google.com/ books?id=v1DBKE7-UrYCandamp; printsec $=$ frontcoverandamp; $\mathrm{dq}=$ in title:Networks+of+the+Brainand amp;hl=andamp;cd=landamp;sour ce=gbs_api

Sporns, O., and Honey, C. J. (2006). Small worlds inside big brains. Proc. Natl. Acad. Sci. U.S.A. 103, 19219-19220. doi: 10.1073/pnas. 0609523103

Sporns, O., Honey, C. J., and Kötter, R. (2007). Identification and classification of hubs in brain networks. PLoS ONE 2:e1049. doi: 10.1371/journal.pone.0001049

Stephan, K. E., Baldeweg, T., and Friston, K. J. (2006). Synaptic plasticity and dysconnection in schizophrenia. Biol. Psychiatry 59, 929-939. doi: 10.1016/j.biopsych. 2005.10.005

Stephan, K. E., Friston, K. J., and Frith, C. D. (2009). Dysconnection in schizophrenia: from abnormal synaptic plasticity to failures of self-monitoring. Schizophr. Bull. 35, 509-527. doi: 10.1093/schbul/sbn 176

Sumich, A., Chitnis, X. A., Fannon, D. G., O'Ceallaigh, S., Doku, V. C., Faldrowicz, A., et al. (2005). Unreality symptoms and volumetric measures of Heschl's gyrus and planum temporal in first-episode psychosis. Biol. Psychiatry 57, 947-950. doi: 10.1016/j.biopsych. 2004.12.041

Tanaka, S. (2006). Dopaminergic control of working memory and its relevance to schizophrenia: a circuit dynamics perspective. Neuroscience 139, 153-171. doi: 10.1016/j.neuro science.2005.08.070

van den Heuvel, M. P., Mandl, R. C. W., Stam, C. J., Kahn, R. S., and Hulshoff Pol, H. E. (2010). Aberrant frontal and temporal complex network structure in schizophrenia: a graph theoretical analysis. J. Neurosci. 30, 15915-15926. doi: 10.1523/ JNEUROSCI.2874-10.2010

van den Heuvel, M. P., and Sporns, O. (2011). Rich-club organization of the human connectome. J. Neurosci. 31, 15775-15786. doi: 10.1523/JNEUROSCI.3539-11.2011 van Wijk, B. C. M., Stam, C. J., and Daffertshofer, A. (2010). Comparing brain networks of different size and connectivity density using graph theory. PLOS ONE 5:e13701. doi: 10.1371/journal. pone.0013701

Wang, Q., Su, T.-P., Zhou, Y., Chou, K.-H., Chen, I.-Y., Jiang, T., et al. (2011). Anatomical insights into disrupted small-world networks in schizophrenia. NeuroImage 59, 1085-1093. doi: 10.1016/j.neuro image.2011.09.035

Watts, D. J., and Strogatz, S. H. (1998). Collective dynamics of "smallworld" networks. Nature 393 440-442. doi: 10.1038/30918

Wechsler, D. (1991). Wechsler Intelligence Scale for Children. San Antonio, TX: The Psychological Corporation.
Wechsler, D. (1997). Wechsler Adult Intelligence Scale. San Antonio, TX: Administration and Scoring Manual.

Yu, Q., Allen, E., Sui, J., Arbabshirani, M., Pearlson, G., and Calhoun, V. (2012). Brain connectivity networks in schizophrenia underlying resting state functional magnetic resonance imaging. Curr. Top. Med. Chem. 12, 2415-2425. doi: 10.2174/ 156802612805289890

Yu, Q., Plis, S. M., Erhardt, E. B. Allen, E. A., Sui, J., Kiehl, K. A. et al. (2011a). Modular organization of functional network connectivity in healthy controls and patients with schizophrenia during the resting state. Front. Syst. Neurosci. 5:103 doi: 10.3389/fnsys.2011.00103

Yu, Q., Sui, J., Rachakonda, S., He, H., Gruner, W., Pearlson, G. et al. (2011b). Altered topological properties of functional network connectivity in schizophrenia during resting state : a small-world brain network study. PLoS ONE 6:e25423. doi: 10.1371/journal. pone. 0025423

Yu, Q., Sui, J., Liu, J., Plis, S. M., Kiehl, K. A., Pearlson, G., et al. (2013). Disrupted correlation between low frequency power and connectivity strength of resting state brain networks in schizophrenia. Schizophr. Res. 143, 165-171. doi: 10.1016/j. schres.2012.11.001

Zalesky, A., Fornito, A., Harding, I. H., Cocchi, L., Yücel, M., Pantelis, C., et al. (2010a). Wholebrain anatomical networks: does the choice of nodes matter? Neuroimage 50, 970-983. doi: 10.1016/j.neuroimage.2009.12.027

Zalesky, A., Fornito, A., Seal, M. L., Cocchi, L., Westin, C. F., Bullmore, E. T., et al. (2010b).
Disrupted axonal fiber connectivity in schizophrenia. Biol. Psychiatry 69, 80-89. doi: $\quad$ 10.1016/j.biopsych.2010. 08.022

Zhang, Y., Lin, L., Lin, C.-P., Zhou, Y., Chou, K.-H., Lo, C.-Y., et al. (2012). Abnormal topological organization of structural brain networks in schizophrenia. Schizophr. Res. 141, 109-118. doi: 10.1016/j.schres.2012. 08.021

Conflict of Interest Statement: The authors declare that the research was conducted in the absence of any commercial or financial relationships that could be construed as a potential conflict of interest.

Received: 08 April 2013; accepted: 09 July 2013; published online: 05 September 2013.

Citation: Ottet M-C, Schaer M, Debbané $M$, Cammoun L, Thiran J-P and Eliez $S$ (2013) Graph theory reveals dysconnected hubs in 22q11DS and altered nodal efficiency in patients with hallucinations. Front. Hum. Neurosci. 7:402. doi: 10.3389/fnhum.2013.00402

This article was submitted to the journal Frontiers in Human Neuroscience.

Copyright (C) 2013 Ottet, Schaer, Debbané, Cammoun, Thiran and Eliez. This is an open-access article distributed under the terms of the Creative Commons Attribution License (CC BY). The use, distribution or reproduction in other forums is permitted, provided the original author(s) or licensor are credited and that the original publication in this journal is cited, in accordance with accepted academic practice. No use, distribution or reproduction is permitted which does not comply with these terms. 